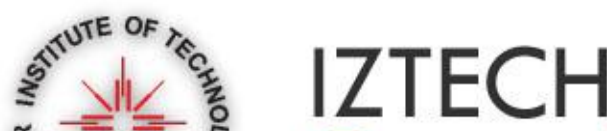 \\ Open Access Articles
}

Formation of Pseudoisocyanine J-Aggregates in Poly(vinyl alcohol) Fibers by Electrospinning

The IZTECH Faculty has made this article openly available. Please share how this access benefits you. Your story matters.

\begin{tabular}{|l|l|}
\hline Citation & $\begin{array}{l}\text { Demir, M, Özen, B, and Özçelik, S, "Formation of } \\
\text { Pseudoisocyanine J-Aggregates in Poly(vinyl alcohol) Fibers } \\
\text { by Electrospinning" Journal of Physical Chemistry B @ 2009 } \\
\text { Elsevier }\end{array}$ \\
\hline As Published & $10.1021 /$ jp902380n \\
\hline Publisher & Elsevier \\
\hline Version & PUBLISHED ARTICLE \\
\hline Accessed & FRI JULY 5 14:41:52 GMT 2013 \\
\hline Citable Link & http://hdl.handle.net/11147/ \\
\hline Terms of Use & $\begin{array}{l}\text { Article is made available in accordance with the publisher's } \\
\text { policy and may be subject to Turkish copyright law. Please } \\
\text { refer to the publisher's site for terms of use. }\end{array}$ \\
\hline Detailed Terms & \\
\hline
\end{tabular}




\title{
ARTICLES
}

\section{Formation of Pseudoisocyanine J-Aggregates in Poly(vinyl alcohol) Fibers by Electrospinning}

\author{
Mustafa M. Demir,* Bengisu Özen, and Serdar Özçelik* \\ Department of Chemistry, İzmir Institute of Technology, Gülbahçe Köyü, Urla 35430 Izmir, Turkey
}

Received: March 17, 2009; Revised Manuscript Received: July 3, 2009

\begin{abstract}
Submicrometer diameter, light emitting fibers of poly(vinyl alcohol) (PVA) doped with pseudoisocyanine (1,1'-diethyl-2,2'-cyanine bromide, PIC) dye were prepared by electrospinning. A horizontal setup was employed with a stationary collector consisting of two parallel-positioned metal strips separated by a void gap. Formation of uniaxially aligned and randomly deposited fibers in electrospun films was confirmed by microscopy. Photoluminescence (PL) spectroscopy is used to evaluate spectral properties of both types of fibers doped with PIC. While PIC molecules were individually dispersed in PVA solution, they assemble into J-aggregates upon electrospinning when the weight fraction of PIC molecules is above $2.5 \mathrm{wt} \%$. The formation of $\mathrm{J}$-aggregates was observed in both randomly deposited and uniaxially aligned electrospun fibers. Moreover, the fibers aligned uniaxially showed a high degree of polarized emission $\left(\mathrm{PL}_{\mid} / \mathrm{PL}_{\perp}=10\right)$, arising from the orientation of J-aggregates along the fiber axis. On the other hand, isotropic emission of J-aggregates was observed from the fibers deposited randomly. As a conclusion, electrospinning was found to be an efficient and a practical method to form highly oriented J-aggregates dispersed into polymer fibers. To the best of our knowledge, it is the first time formation of J-aggregates (a bottom-up approach) and electrospinning (a topdown approach) is successfully combined.
\end{abstract}

\section{Introduction}

The properties of supramolecular materials are governed by collective rather than individual behavior of molecules. In turn, the collective properties are strongly mediated by intermolecular interactions and microscopic molecular packing, that is, spatial arrangements of molecules through electrostatic forces. Molecular interactions and arrangements can be controlled by concentration of molecules, environmental conditions (phase, solvent, $\mathrm{pH}$, temperature, ionic strength, etc.) and structure of chromophore. Alignment of the ordered supramolecular structures like J-aggregates through the self-organization of small molecules is considered to be the key to advanced functional organic materials for electronics and photonics. ${ }^{1}$ Pseudoisocyanines (PIC) are a peculiar class of aggregating dyes, ${ }^{2}$ which form J-aggregates in various arrangements from one-dimensional chain or ribbon-like structures to three-dimensional cylindrical structures. ${ }^{3}$ J-aggregates exhibit a characteristic red-shifted intense and sharp transition that is lower than the transition energy of the monomer. This new transition is associated with resonance fluorescence due to the formation of exciton states. The presence of the J-band is usually ascribed to stacking of monomer molecules and corresponding ordered transition dipole moments that lead to delocalization of excitons over an aggregate domain. ${ }^{4}$ Since the internal organization of molecular structure determines the performance, it is important to develop techniques for fabrication of structures at a molecular level. A number of methods have been developed to form and to align

* Corresponding authors. Phone: +90 23275075 11. Fax: +90 232 75075 09. E-mail: mdemir@iyte.edu.tr and serdarozcelik@iyte.edu.tr. the aggregates such as vertical spin coating, ${ }^{5-7}$ flow of solution, ${ }^{8}$ evaporation of solution, ${ }^{9}$ and magnetic fields. ${ }^{10}$ Here, we report a new method to prepare highly ordered J-aggregates by the electrospinning process within submicrometer diameter poly(vinyl alcohol) (PVA) fibers. The formation and alignment of J-aggregates of dye molecules via electrospinning has not yet been reported according to the best of our knowledge.

Electrospinning has proven to be an efficient method for generating nanometer scale polymeric fibers out of polymer solutions and melts. Typically, a high potential difference is created between a droplet of polymer solution hold in a thin glass tube (a reservoir) and a conductive grounded sheet at ambient conditions. When electrical force overcomes the surface tension of the droplet, a thin jet stream of polymer solution is ejected from the nozzle of the tube. Evaporation of solvent and continuous stretching of the electrostatic force in the charged jet result in the formation of continuous fibers. Eventually, a polymeric thin film consisting of a nonwoven fiber mat appears on the surface of the grounded conductive sheet within minutes. In earlier works, fibers made from more than 30 different types of polymers have been obtained by electrospinning. ${ }^{11}$ The fibers are usually obtained as nonwoven mats; however, they can also be readily prepared as aligned arrays at the macroscopic level by using a collector consisting of two grounded conductive metal films separated by a millimeter-length air gap. ${ }^{12,13}$ The electrical field applied during the spinning process enforces to orient the polymer chains at the molecular level ${ }^{14-16}$ and as well as 1-dimensional constituent units in spinning solution such as wires, ${ }^{17}$ tubes, ${ }^{18}$ etc. Consequently, electrospinning is not only a robust technique to produce polymeric nanofibers but also a 
method to fabricate well-ordered nanostructured materials for various applications. ${ }^{17-23}$ In our previous work, ${ }^{19}$ we reported controlled spontaneous emission of CdSe nanoparticles dispersed in an electrospun mat based on the segmented poly(carbonate urethane) copolymer. Elongational flow of the electrospinning process orients the copolymer domains associated with the particles. New multiple sharp lines were observed in the emission spectra of the composite mats, which were not observed in the spectra of CdSe particles in toluene. Zusmann et al. ${ }^{17}$ reported polarized photoluminescence of $\mathrm{CdS}$ quantum wires oriented within electrospun PEO fibers. Chae et al. ${ }^{23}$ proposed electrospinning of a $\pi$-conjugating diaryl-substituted polyacetylene, which forms a porous honeycomb network structure resulting in optical anisotropy of a strong emission perpendicular to the fiber axis. In this work, we present the formation of J-aggregates of 1,1'-diethyl-2,2'-cyanine bromide (PIC) upon electrospinning. The orientation of the aggregates in both uniaxially aligned and randomly deposited PVA fibers is important in terms of functional properties of materials. The structural difference in the constituent fibers controls the polarization state of photoluminescence emission. It has to be noted that the word "orientation" refers to organization of the J-aggregates within PVA fibers and the word "alignment" associates with the macroscopic arrangement of the fibers.

\section{Experimental Section}

1,1'-Diethyl-2,2'-cyanine bromide, PIC from Hayashibara, Japan, and poly(vinyl alcohol) (PVA, 87-90\% hydrolyzed and $30-70 \mathrm{~kg} \cdot \mathrm{mol}^{-1}$ ) were purchased from Sigma-Aldrich, USA. The dye/polymer solution was prepared as follows. ${ }^{5,6}$ In a typical procedure, $80 \mathrm{mg}$ of PVA was dissolved into $2 \mathrm{~mL}$ of distilled water at $\sim 100^{\circ} \mathrm{C}$. A homogeneous solution including a desired amount of PIC in $2 \mathrm{~mL}$ of methanol was added dropwise into the PVA solution (2.5-15 wt. \%) under stirring. The methanol fraction of the solution was evaporated, and the resulting aqueous dye/polymer solution was subjected to electrospinning at $35^{\circ} \mathrm{C}$ in a horizontal direction in a glass chamber. The details of the spinning process were given elsewhere. ${ }^{24}$ Instrumental parameters, which were a spinning distance of $6 \mathrm{~cm}$ and an applied potential difference of $1.6 \mathrm{kV} \cdot \mathrm{cm}^{-1}$, and the void gap between two metal strips $(1.0 \mathrm{~mm})$ were kept constant for all experiments. Evaporation of solvent during the continuous electrical force results in nucleation and orientation of $\mathrm{J}$ aggregates in the PVA fibers. Absorption and PL spectra were obtained by a Varian Eclips spectrofluorometer equipped with polarizers. Infrared spectra were registered with a Perkin-Elmer Spectrum 100 Fourier Transform Infrared Spectrometer. Polarization of the incident infrared radiation was achieved with a $\mathrm{ZnSe}$ polarizer. Images of scanning electron (acceleration voltage is $3 \mathrm{kV}$ ) and confocal microscopes (laser excitation at $488 \mathrm{~nm}$ ) were collected by Phillips XL-30S FEG and Andor Revolution laser confocal system (lateral resolution of $250 \mathrm{~nm}$ and axial resolution of $700 \mathrm{~nm}$ ), respectively. Atomic force microscope (AFM) investigation was performed using Digital Instruments (Nanoscope IIIa) employing the tapping mode in air for the spin-coated films.

\section{Results and Discussion}

PIC/PVA solutions were electrospun using a conventional horizontal spinning setup where the collector was designed by two parallel stainless steel strips separated by a void gap. An overview of the collector system was imaged by a scanning electron microscope (SEM) shown in panel a of Figure 1. An electrospun mat obtained on the collector is not homogeneous
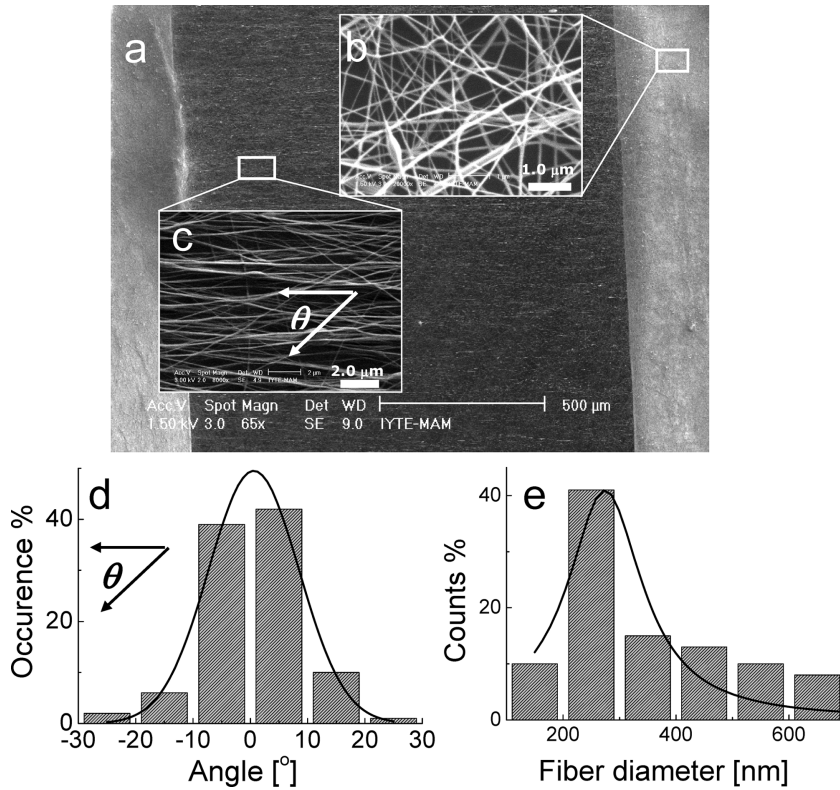

Figure 1. (a) SEM micrograph of the collector and electrospun PVA fibers. The collector consists of two parallel stainless steel strips separated by a $1 \mathrm{~mm}$ void gap. The mat obtained on this type of collector contains both randomly deposited and uniaxially aligned fibers. Scale bar is $0.5 \mathrm{~mm}$. (b) SEM image of the electrospun fibers collected on the surface of metal strips shows randomly deposited fibers. Scale bar is $1.0 \mu \mathrm{m}$. (c) The fibers are aligned uniaxially across the void gap. Scale bar is $2.0 \mu \mathrm{m}$. (d) Histogram of the angle $(\theta)$ between the long axis of fibers and the normal of the long edge of the metal electrodes. The histogram was prepared based on measurements of more than 100 test fibers. (e) The mean diameter of fibers is around 0.37 $\mu \mathrm{m}$ under an electrical field of $1.6 \mathrm{kV} \cdot \mathrm{cm}^{-1}$.

in nature. On the metal strips, PVA fibers were randomly deposited, and they have no preferential direction (panel b). However, PVA fibers formed across the gap appeared to be aligned perpendicular to the long edge of the strips (panel c). Hence, electrospun film consisting of both isotropic and anisotropic fiber populations was obtained by spinning of the same solution in a single run. The degree of alignment of fibers was assessed by statistical treatment of the angle $(\theta)$ between the fibers and normal of the metal strips. The majority of fibers fall in the range of $0^{\circ}-10^{\circ}$ (panel d of Figure 1), suggesting a high degree of alignment. The diameter of the resulting fibers exhibits a Lorentzian type of distribution centered at $0.37 \pm$ $0.08 \mu \mathrm{m}$ (panel e of Figure 1). The density of fibers on the metal strips is higher as compared to the ones in the void gap because as-spun fibers are highly charged so that they induce opposite charges on the surface of electrodes, which further attract the fibers through the metal electrodes.

Figure 2 presents absorption spectra of PIC/PVA solutions in a mixture of water and methanol at $35^{\circ} \mathrm{C}$. The spectra of various concentrations ranging from 1.7 to $14.1 \mathrm{mM}$ exhibit the absorbance of a PIC monomer (broadband) at $522 \mathrm{~nm}$. Not surprisingly, the absorbance increases with the concentration of PIC, and it exceeds the upper limit of the spectrophotometer at $14.1 \mathrm{mM}$ even though the thinnest cuvette (path length: 1 $\mathrm{mm}$ ) was employed. This result proves that PIC molecules are individually dispersed in solution, and the spinning solution is initially free of J-aggregates. The monomeric dye and polymer solutions were subjected to electrospinning to investigate the effect of the electrospinning process on the aggregation behavior of PIC molecules. In this regard, three different weight percentages of PIC $(2.5,7.0$, and $15.0 \mathrm{wt} \%)$ in aqueous PVA solutions were prepared and electrospun at $35{ }^{\circ} \mathrm{C}$. As SEM images 


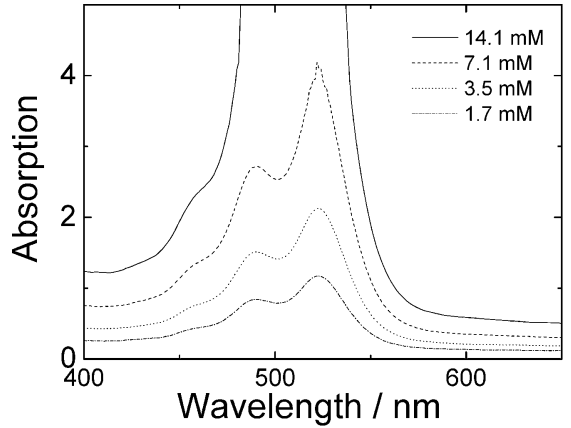<smiles>CCN1C(=Cc2ccc3ccccc3[n+]2CC)C=Cc2ccc(Br)cc21</smiles>

Figure 2. (a) Absorption spectra of PIC in aqueous PVA solutions of different weight percents at $35^{\circ} \mathrm{C}$ and the chemical structure of PIC. No J-band is observed. (The path length of the cuvette is $1.0 \mathrm{~mm}$.)

indicate, the diameter of the resulting fibers is comparable with the wavelength of visible light, hence a significant amount of optical scattering by the PIC/PVA fibers (an opaque mat) was observed. Therefore, one can focus on reflection and photoluminescence spectroscopies (PL) for the examination of spectral properties. Figure 3a displays reflection spectra of the cast film and the electrospun fibers prepared from the same PIC/PVA solution (15:85 by mass). The cast film shows a very broad featureless band between 400 and $600 \mathrm{~nm}$ without indicating a clear signal. On the other hand, the spectra of the electrospun mats exhibit a sharp band at $589 \mathrm{~nm}$ and two broad bands at 525 and $490 \mathrm{~nm}$. The sharp band assigned as the J-band is an unambiguous evidence of the presence of J-aggregates within the fibers. The J-band is usually very sharp in homogeneous samples such as aqueous solutions. ${ }^{25}$ In polymeric films, it is also very sharp; however, J-aggregates interact with matrix. ${ }^{6}$ In addition, the spectral position of the J-band of PIC varies with medium and as well as preparation methods. In PVA films, the J-band usually appears around $580 \mathrm{~nm}$, whereas in aqueous solution the position of the J-band is around $570 \mathrm{~nm} .{ }^{6,25}$ The difference in spectral position of the J-band is generally attributed to the effect of medium on the formation of $\mathrm{J}$ aggregates. For the two bands at 525 and $490 \mathrm{~nm}$ in the spectrum, one can consider the presence of monomeric PIC. However, our previous studies showed that these bands are not originated from the monomeric PIC but may be due to the upper exciton states of the J-aggregates ${ }^{26}$ and/or some intermediate aggregates that may lead nucleation of J-aggregates. ${ }^{27}$

Figure $3 \mathrm{~b}$ shows normalized photoluminescence spectra of the randomly deposited PIC/PVA fibers and of the corresponding cast film prepared from the same electrospinning solution. At the lowest weight percent, a broad PL band positioned around $550 \mathrm{~nm}$ appears and is attributed to the presence of individual PIC molecules dispersed in the electrospun fibers. Previously, it was shown that PIC dissolved in glycerol exhibits a band around $530 \mathrm{~nm}$, assigned as monomers in solution at room temperature. ${ }^{28}$ Approximately a $20 \mathrm{~nm}$ red-shift of the monomer band in our result can be due to the environmental change from solution to the solid phase. A shoulder at $620 \mathrm{~nm}$ in the spectrum of the electrospun mat with the lowest PIC weight percent is apparent, and its nature will be discussed below. As the weight fraction of PIC increases, new features in the spectra were observed. The broadband at $550 \mathrm{~nm}$ assigned to the monomer
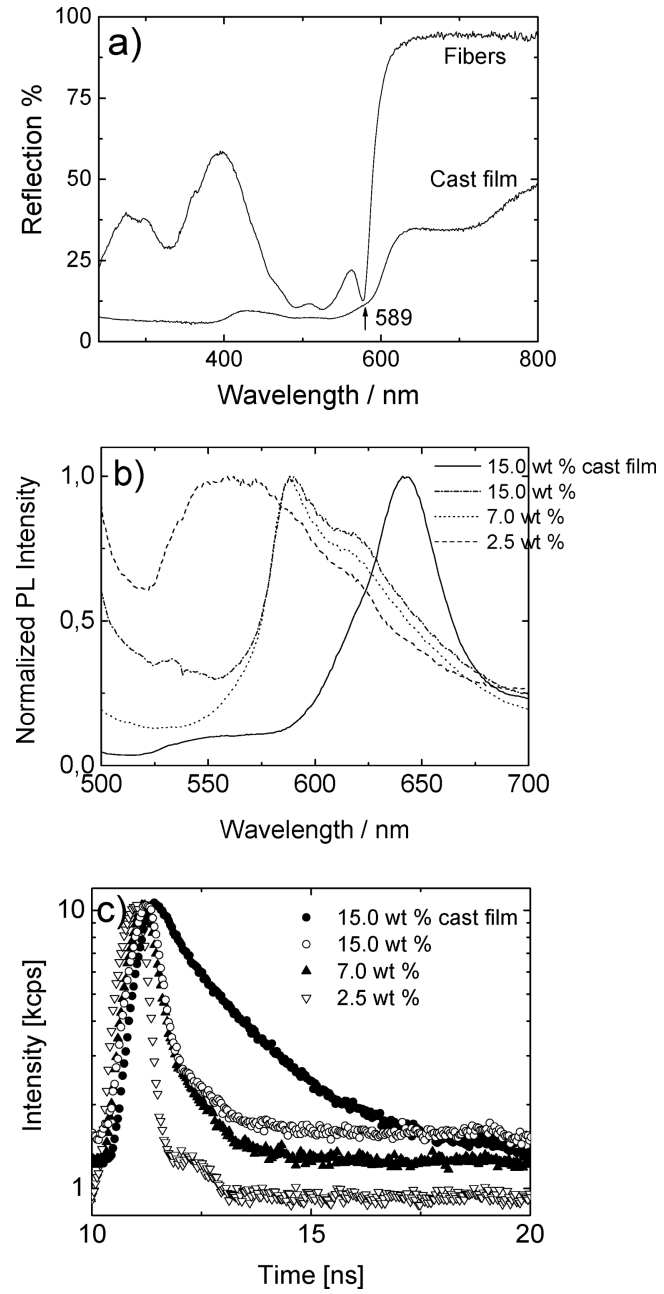

Figure 3. (a) Reflection spectra of the electrospun PIC/PVA fibers and the cast film. The weight percent of PIC in PVA is 15 in both cases. (b) The effect of weight fraction on photoluminescence spectra of PIC obtained from randomly deposited PVA fibers. The spectra are normalized with respect to maxima in each spectrum. (c) Decay curves of the fibers excited at $450 \mathrm{~nm}$. The values are $0.17 \mathrm{~ns}$ and $0.70 \mathrm{~ns}$ (2.5 wt \%), $0.14 \mathrm{~ns}$ and $0.80 \mathrm{~ns}(7.0 \mathrm{wt} \%), 0.13 \mathrm{~ns}$ and $0.89 \mathrm{~ns}(15.0$ wt \%), and $0.29 \mathrm{~ns}$ and $1.65 \mathrm{~ns}$ (15.0 wt \% for cast film).

substantially vanishes, and a new one at $589 \mathrm{~nm}$ appears with a shoulder centered at $620 \mathrm{~nm}$. The band at $589 \mathrm{~nm}$ is a typical J-band indicating the formation of PIC J-aggregates in electrospun PVA fibers that was initially absent in the spinning solution. It is a broadband due to structural disorders and inhomogeneities within the J-aggregates dispersed throughout the fiber volume. The fluorescence (PL) spectrum of the J-band is generally very sharp in homogeneous solution and in thin PVA films. ${ }^{5}$ This sharp feature is originated from structures with lower amount of disorders in J-aggregates. However, broader J-bands in PL spectra of J-aggregates adsorbed on surfaces such as colloid are frequently observed. This observation is due to the structural disorders and energetic heterogeneities. ${ }^{29}$ As observed in this study, the broader J-band is a common feature and can be reduced by controlling experimental conditions which may force the formation of the J-aggregate with less structural disorders.

The nature of the shoulder at $620 \mathrm{~nm}$ is elusive according to the literature. Muenter et al. ${ }^{29}$ proposed that the emission band at $620 \mathrm{~nm}$ arises from a dimeric state of PIC in aqueous solution; meanwhile, Lebedenko et al. ${ }^{30}$ recently showed that this shoulder originates as a result of a transition taking place in PIC crystals 
in powder. However, we propose that the appearance of the shoulder may be attributed to the presence of different types of stacking faults or defects in the molecular arrangement of dye molecules. If it is the case, the intensity of emission arising from the defects ought to increase as the amount of dye content increases. Indeed, the intensity of emission at $620 \mathrm{~nm}$ with respect to that of the J-band is pronounced as the weight percent of PIC molecules increases (comparing the normalized band intensities when the weight percents are 7.0 and 15.0 in Figure $3 b$ ). We interpret this observation as evidence of defects formed within the structure of the J-aggregate dispersed into PVA fibers. The density of defects varies with alignment of the fiber assemblies. The intensity of the band at $620 \mathrm{~nm}$ is lower in uniaxially aligned fibers as compared to the one of randomly deposited fibers (Figure 1 of Supporting Information). Jaggregates with a reduced number of defects are obtained when the fibers were produced within the air gap due to high elongational stress between the sharp electrodes. Another scenario that may lead to the appearance of the band at $620 \mathrm{~nm}$ can be due to an electronic transition originating from spatially different sites of J-aggregates. To support this hypothesis, a new set of systematic experiments have to be designed and performed.

As a control experiment, a cast film was prepared from the same electrospinning solution of $15.0 \mathrm{wt} \%$ at $35{ }^{\circ} \mathrm{C}$. The PL spectrum of the cast film exhibits an intense emission at 640 $\mathrm{nm}$. Molecular arrangement and morphology of the dye and/or the dye/polymer structure that reflects this emission is unknown and is under investigation. A weak emission at $550 \mathrm{~nm}$ concurrences with the emission of individual PIC and a vague shoulder at $620 \mathrm{~nm}$ are also present. This result clearly demonstrates the significance of the electrospinning process in the formation of J-aggregates. While the dye molecules form an energetically more stable and unidentified structure by filmcasting, the electrospining process yields a supramolecular structure with an ordered molecular arrangement (J-aggregates).

Figure $3 \mathrm{c}$ shows PL decay curves of the electrospun mats and of the cast film when excited at $450 \mathrm{~nm}$. The PL lifetimes were found to depend on the weight fraction of PIC. Biexponential decay dynamics were observed in all cases. This is a result of a kinetic parametrization and may originate from the distributed nature of the J-aggregates. The lifetime of the short component becomes systematically faster $(0.17$ down to 0.13 ns) when the weight fraction of PIC is increased from 2.5 to $15.0 \mathrm{wt} \%$, respectively. In contrast, the long component of the decay signals prolonged from 0.71 to $0.90 \mathrm{~ns}$. The reduction in the short component is indicative of a superradiant state, which is important evidence for the formation of the J-aggregate in the electrospun fibers. The cast film also exhibited a biexponential decay curve with characteristic times of 0.29 and 1.65 ns. The emission of the cast film is further red-shifted and has a longer lifetime component than the J-aggregates in electrospun fibers even though the weight fraction of PIC remains unchanged. Therefore, the existence of J-aggregates in the cast film is ruled out. Transitions between the excitonic states of J-aggregates, in general, are expected to be faster because the lifetime of excitonic states is inversely proportional with the number of individual molecules in J-aggregates. ${ }^{26,29}$ However, a formation of excimer-type structures is plausible and worth investigating, but it is not in the scope of this study.

On the basis of the comparative results of the electrospun mats and the cast film given in Figure 3, it must be noted that the internal structure (packing of PIC molecules) of the cast film differs strongly from that of the electrospun film. As evidence, the PL spectrum displays an intense band at $640 \mathrm{~nm}$.

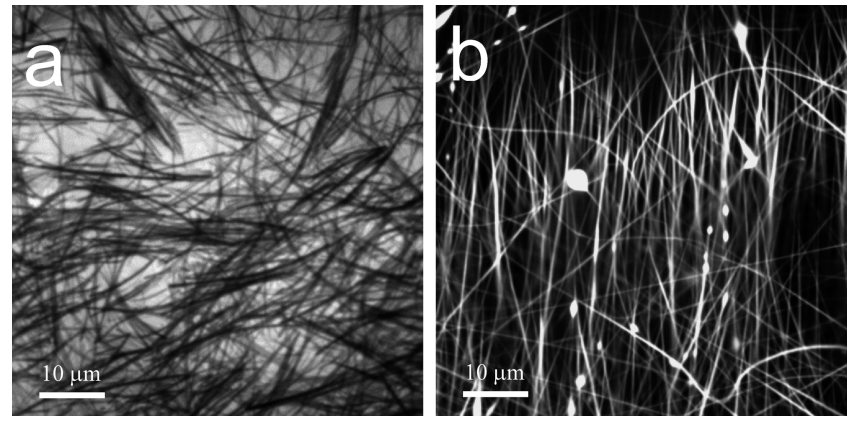

Figure 4. Fluorescence image of (a) the rodlike PIC crystallite and (b) the PIC-doped $(7.5 \mathrm{wt} \%)$ electrospun PVA fibers imaged by a confocal microscope. The chromophore aggregates are distributed homogeneously over the entire fiber volume. The areas of $50 \times 50$ $\mu \mathrm{m}^{2}$ are being evenly illuminated. The wavelength of the laser is 488 $\mathrm{nm}$. A dichroic filter for GFP and an emission filter of LP500 are used for confocal imaging.

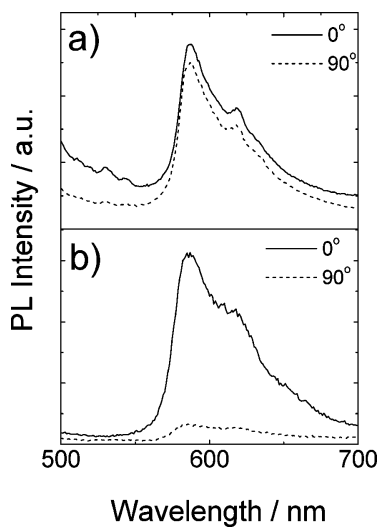

Figure 5. Polarized PL spectra of J-aggregates in (a) randomly deposited and (b) uniaxially aligned fibers registered by using polarized light parallel and perpendicular to the fiber direction.

This result is consistent with the literature. ${ }^{31}$ Moreover, the decay components of the cast film are longer, which is another proof supporting the absence of J-aggregates in the cast film. The results obtained by PL spectroscopy and dynamics validate the observation of the sharp band at $589 \mathrm{~nm}$ in the reflection spectrum (Figure $3 \mathrm{a}$ ) originating from the J-aggregates.

The spectral and dynamic dissimilarities between cast and electrospun films are considered to be a reflection of the structural difference that is confirmed by confocal laser scanning microscopy. Figure 4 presents $50 \times 50 \mu \mathrm{m}^{2}$ fluorescence images of the cast film (panel a) and of the electrospun film (panel b). In the first image, rodlike objects with diameters of about 0.3 $\mu \mathrm{m}$ and lengths of $20 \mu \mathrm{m}$ have been observed. The rods were mainly straight or slightly bent. These rodlike objects are supposed to be crystallites of either dye aggregates and/or the composite structure of dye and matrix PVA molecules. Panel b shows the confocal image of uniaxially aligned PVA fibers deposited in the void gap. The fluorescence of electospun fibers points out that the dye aggregates are uniformly distributed over the fiber volume, although the fibers at the top most layer seem to be distorted which unavoidably takes place during sample preparation. The alignment of the fibers at sublayers was observed clearly.

Figure 5 illustrates the polarized PL spectra of the electrospun fibers obtained from PIC/PVA (15:85 by wt \%) solution. The features of the PL spectra obtained from randomly deposited and aligned fibers are strikingly different. For randomly deposited fibers, both spectra obtained when light was polarized parallel and perpendicular to the direction of fibers were similar 


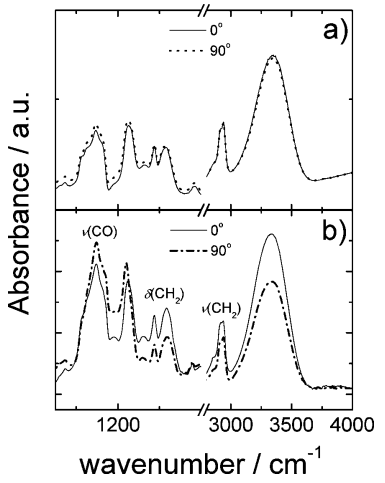

Figure 6. Polarized FTIR spectra of (a) randomly deposited and (b) uniaxially aligned PVA fibers electrospun at $1.6 \mathrm{kV} \cdot \mathrm{cm}^{-1}$. The difference in absorbance could be evidence of chain alignment within the fibers.

in terms of spectral shape and intensity (Figure 5a). Since the fibers deposited on the metal strips have no preferential directions, the electrospun mat exhibited isotropic PL behavior. Electrospun films have a multilayer structure consisting of many fibers deposited randomly; therefore, the orientation of $\mathrm{J}$ aggregates in the bulk electrospun mat is averaged out. In contrast, a very strong polarized emission was observed for the aligned fibers (Figure 5b). The PL spectrum measured with light that was polarized parallel to the fiber direction showed an intense J-band at $589 \mathrm{~nm}$. Rotating the polarizer $90^{\circ}$ (perpendicular to the fiber direction) reduced the PL intensity of the J-band dramatically. The estimated ratio of $\mathrm{PL}_{\mid} / \mathrm{PL}_{\perp}$ of the J-band is nearly 10. This polarization dependence strongly indicates that the orientation of constituent PIC molecules forming J-aggregates occurs on the direction of PVA fibers.

The morphology in terms of molecular orientation of PVA under the electrical field was also investigated by polarized FTIR spectroscopy where an IR beam probe was polarized parallel and perpendicular to the fiber axis. Figure 6 shows the polarized FTIR spectra of randomly deposited and uniaxially aligned fibers of PIC/PVA blend. The FTIR spectra of PVA fibers are in good agreement with the literature. ${ }^{32}$ Parallel and perpendicularly polarized spectra of randomly deposited fibers are found to be alike. The positions and intensities of the bands are almost identical indicating isotropic feature of randomly oriented fibers. However, in the aligned fibers, parallel and perpendicularly polarized FTIR spectra in terms of absorbance are remarkably different. Although the bands appear at the same wavenumbers, their intensities vary depending on the polarization of the incident IR beam. This dichroic behavior can be correlated with the orientation of the PVA backbone within electrospun fibers. PVA is obtained by hydrolysis of poly(vinyl acetate), and therefore the backbone of PVA is composed of carbon atoms. Depending on the degree of hydrolysis, alternating carbon atoms possess either hydroxyl or acetate groups. Stretching of $\mathrm{CH}_{2}$ groups on the backbone at $2942 \mathrm{~cm}^{-1}$ is parallel to the backbone of the chain and is called backbone vibration. On the other hand, the stretching between carbon atoms on the backbone and the pendant oxygen of hydroxyl or acetate groups (CO) at 1097 $\mathrm{cm}^{-1}$ is considered as pendant vibration. The vibration of the pendant group is perpendicular to that of the backbone one. The comparison of absorbance of the backbone and pendant vibrations in polarized FTIR spectra provides a valuable insight to understanding of internal orientation of PVA chains within PVA fibers. At $2942 \mathrm{~cm}^{-1}$, the absorbance of backbone vibration is higher when the incident IR beam is polarized parallel to the fiber axis. However, at $1097 \mathrm{~cm}^{-1}$, the pendant bond vibration becomes more pronounced with respect to backbone vibrations when the beam is perpendicularly polarized. A higher number of $\mathrm{CO}$ bonds is coupled with the perpendicularly polarized IR beam, giving a more intense signal. The difference in absorbance intensities of backbone and pendant group vibrations between parallel and perpendicular polarization states is obvious evidence for the molecular orientation of PVA along the fiber axis. The degree of uniaxial orientation of PVA chains by electrospinning can be quantified by the dichroic ratio. ${ }^{16}$ The dichroic ratio $(R)$ is defined by the formula $R=A_{\|} / A_{\perp}$, where $A_{\|}$is infrared absorbance of the parallel component and $A_{\perp}$ is that of the perpendicular component. For a randomly oriented sample, $R$ is equal to 1 , and for a perfectly uniaxially oriented sample, $R$ is equal to infinity. The dichroic ratios for the bands at 2942 and $1097 \mathrm{~cm}^{-1}$ are 1.4 and 0.8 , respectively. It has to be noted that the dichroic ratio of electrospun PVA fibers is lower than those reported in the literature for polyoxymethylene ${ }^{16,33}$ and polyethyleneoxide fibers. ${ }^{15}$ The lower degree of orientation of PVA molecules, compared to other macromolecules in the literature, may be related to strong inter- and intramolecular interactions.

Elongation within the charged jet stream is an important step in the electrospinning process, and its rate has been reported as high as $10^{4} \mathrm{~s}^{-1} \cdot{ }^{14,34}$ The rapid formation of the structure taking place on a millisecond scale is shorter than the time required to organize an ordered structure of polymer chains. However, the formation of well-ordered supramolecular structures from dye molecules by electrical force should readily occur within this time scale based on our experimental results. Dye molecules individually dissolved (monomers dispersed in solution) in the spinning solution undergo polymerization of J-aggregates upon electrospinning. In fact, the elongational force during electrospinning gains particular importance when the components of spinning solution are considered. Water molecules as the medium of the solution possess very high dipole moments, which lead to a strong tendency for orientation under the electrical field. Moreover, the presence of dye molecules, which are in fact salts, further increases the ionic strength of the spinning solution. PVA contains the $\mathrm{OH}$ group on the backbone as the pendant group that leads to inter/intramolecular hydrogen bonding which is directed bonds that also help to elongate the fibers. A higher rate of elongation flow results in higher stress in the charged jet stream during the electrospinning process. This stress may cause the orientation of constituents at the molecular level that leads to the formation of J-aggregates.

We compared J-aggregates formed in PVA fibers by electrospinning with those fabricated in PVA film by spin-coating techniques. Two different coatings are available to form J-aggregates dispersed in polymer films: vertical spin coating and conventional spin coating. Conventional spin coating fabricates J-aggregates dispersed in polymer films, but no polarized emission is expected due to dispersion of J-aggregates at random. However, examining the samples prepared by vertical spin coatings proved that there is a strong polarized emission (Figure 2 of Supporting Information). The ratio of PL intensities of the J-band is about 9. Hence, both electrospinning and vertical spin coating techniques are capable of producing highly oriented J-aggregates in PVA. Nevertheless, electrospinning has important advantages over the coating method because it is an easier method to prepare and to control the formation of both fibers and dye aggregates. In addition, an electrospun mat can be obtained as free-standing film; in contrast, vertical spin coating needs a substrate. The length of fibers aligned is about submicrometer scale in vertical spin coating while that can be 
extended to millimeter scale in electrospinning that is a key feature for practical applications. Furthermore, a large area of electrospun mat can readily be prepared without a restriction on the size and shape of the sample, which is utterly important for practical applications such as displays that demand stable solid-state materials which can be fulfilled by fabrication of thin films possessing functional chemical units/groups.

\section{Conclusion}

This work combines formation of molecular J-aggregates and fabrication of mesoscale fibers, merging supramolecular chemistry with electrospinning. This unprecedented combination enables us to form a highly oriented one-dimensional (1D) macroscopic structure that may have potential applications in optical devices. Depending on the geometry of grounded electrodes, randomly deposited and uniaxially aligned fiber assemblies can be readily obtained. Regardless of the alignment, J-aggregates of PIC molecules were successfully prepared within PVA fibers by electrospinning. The emission feature of electrospun mats can be controlled by the applied electric field which orients the constituents of the fibers. The former type of fibers exhibited isotropic emission, whereas the latter displayed anisotropic emission which reflects the specific orientation of J-aggregates as emitting species in the aligned nanofibers. Two levels of structural organizations induced by electrospinning are described for the uniaxially aligned fibers. One of them is the alignment of the electrospun PVA fibers at the macroscopic level, which was achieved by separating two sharp metal strips with a void gap. Another type of organization is the orientation of individual PIC molecules to form J-aggregates parallel to the long axis of electrospun fibers. The presence of orientational order at the molecular level inside the macroscopically aligned PVA fibers leads to a hierarchical structure, which is significantly important in optical and electronic assemblies and/or devices. Moreover, a low-cost manufacturing of J-aggregates combined with ease of production of electrospinning makes this composite material very attractive from the point of scaling up. Morphology of J-aggregates and their distribution in PVA fibers are of interest to determine physical structures and to further understand spectral properties of J-aggregates which will pave the way to more scientific and technological applications.

Acknowledgment. The authors thank members of IYTEMAM for microscopy work and S. İçli of Ege University for lifetime measurements. M.M.D. acknowledges the financial support of The Scientific and Technological Research Council of Turkey (TUBITAK) career development project encoded with TBAG-107T795. S.O. thanks the State Planning Organization (DPT) of Turkish Prime Ministry for the financial support.

Supporting Information Available: Comparison of defect density of J-aggregates in randomly deposited and uniaxially aligned fibers, PL spectra of film prepared by vertical spin coating, and AFM image of the film. This material is available free of charge via the Internet at http://pubs.acs.org.

\section{References and Notes}

(1) Steinbeck, C. A.; Ernst, M.; Meier, B. H.; Chmelka, B. F. J. Phys. Chem. C 2008, 112, 2565.

(2) Kirstein, S.; von Berlepsch, H.; Bottcher, C.; Burger, C.; Ouart, A.; Reck, G.; Dahne, S. ChemPhysChem 2000, 1, 146.

(3) Misawa, K.; Kobayashi, T. J-aggregates; World Scientific Publishing: Singapore, 1996.

(4) Ozcelik, S.; Demir, M. M.; Birkan, B. J. Phys. Chem. B 2004, 108, 4679 .

(5) Misawa, K.; Ono, H.; Minoshima, K.; Kobayashi, T. Appl. Phys. Lett. 1993, 63, 577.

(6) Kobayashi, T.; Misawa, K. J. Lumin. 1997, 72-4, 38.

(7) Gulen, D.; Atasoylu, O.; Ozcelik, S. Chem. Phys. 2009, 355, 73.

(8) Schiebe, G. Angew. Chem. 1936, 49, 563.

(9) Takazawa, K.; Kitahama, Y.; Kimura, Y. Chem. Commun. 2004, 2272 .

(10) Shklyarevskiy, I. O.; Boamfa, M. I.; Christianen, P. C. M.; Touhari, F.; van Kempen, H.; Deroover, G.; Callant, P.; Maan, J. C. J. Chem. Phys. 2002, 116, 8407 .

(11) Greiner, A.; Wendorff, J. H. Angew. Chem., Int. Ed. 2007, 46, 5670.

(12) Li, D.; Wang, Y. L.; Xia, Y. N. Adv. Mater. 2004, 16, 361. 913.

(13) Li, D.; Ouyang, G.; McCann, J. T.; Xia, Y. N. Nano Lett. 2005, 5,

(14) Dersch, R.; Liu, T. Q.; Schaper, A. K.; Greiner, A.; Wendorff, J. H. J. Polym. Sci., Part A: Polym. Chem. 2003, 41, 545.

(15) Kakade, M. V.; Givens, S.; Gardner, K.; Lee, K. H.; Chase, D. B.; Rabolt, J. F. J. Am. Chem. Soc. 2007, 129, 2777.

(16) Kongkhlang, T.; Tashiro, K.; Kotaki, M.; Chirachanchai, S. J. Am. Chem. Soc. 2008, 130, 15460.

(17) Bashouti, M.; Salalha, W.; Brumer, M.; Zussman, E.; Lifshitz, E. ChemPhysChem 2006, 7, 102

(18) Ji, J. Y.; Sui, G.; Yu, Y. H.; Liu, Y. X.; Lin, Y. H.; Du, Z. J.; Ryu, S.; Yang, X. P. J. Phys. Chem. C 2009, 113, 4779.

(19) Demir, M. M.; Soyal, D.; Ünlü, C.; Kuş, M.; Özçelik, S. J. Phys. Chem. C 2009, 113, 11273 .

(20) Mathew, G.; Hong, J. P.; Rhee, J. M.; Leo, D. J.; Nah, C. J. Appl. Polym. Sci. 2006, 101, 2017.

(21) Ayres, C.; Bowlin, G. L.; Henderson, S. C.; Taylor, L.; Shultz, J.; Alexander, J.; Telemeco, T. A.; Simpson, D. G. Biomaterials 2006, 27 , 5524

(22) Ra, E. J.; An, K. H.; Kim, K. K.; Jeong, S. Y.; Lee, Y. H. Chem. Phys. Lett. 2005, 413, 188.

(23) Chae, S. K.; Park, H.; Yoon, J.; Lee, C. H.; Ahn, D. J.; Kim, J. M. Adv. Mater. 2007, 19, 521.

(24) Demir, M. M.; Ugur, G.; Gulgun, M. A.; Menceloglu, Y. Z. Macromol. Chem. Phys. 2008, 209, 508.

(25) Herz, A. H. Adv. Colloid Interface Sci. 1977, 8, 237.

(26) Fidder, H.; Terpstra, J.; Wiersma, D. A. J. Chem. Phys. 1991, 94, 6895.

(27) Gulen, D.; Ozcelik, S. "Absorption spectrum of monomeric pseudoisocyanine: A new perspective and its implications for formation and spectral response of J-aggregates in solution and in thin films". 16th International Conference on Dynamical Processes in Excited States of Solids, 2007, Segovia, SPAIN.

(28) Renge, I.; Wild, U. P. J. Phys. Chem. A 1997, 101, 7977.

(29) Muenter, A. A.; Brumbaugh, D. V.; Apolito, J.; Horn, L. A.; Spano, F. C.; Mukamel, S. J. Phys. Chem. 1992, 96, 2783.

(30) Lebedenko, A. N.; Guralchuk, G. Y.; Sorokin, A. V.; Yefimova, S. L.; Malyukin, Y. V. J. Phys. Chem. B 2006, 110, 17772.

(31) von Berlepsch, H.; Bottcher, C. J. Phys. Chem. B 2002, 106, 3146.

(32) Mansur, H. S.; Sadahira, C. M.; Souza, A. N.; Mansur, A. A. P. "FTIR spectroscopy characterization of poly (vinyl alcohol) hydrogel with different hydrolysis degree and chemically crosslinked with glutaraldehyde". Symposium on Nanostructured Biological Materials held at the 5th Meeting of the Brazilian-Materials-Research-Society, 2006, Florianopolis, BRAZIL.

(33) Lu, J. W.; Zhang, Z. P.; Ren, X. Z.; Chen, Y. Z.; Yu, J.; Guo, Z. X. Macromolecules 2008, 41, 3762 .

(34) Doshi, J.; Reneker, D. H. J. Electrost. 1995, 35, 151.

JP902380N 